\title{
Dynamics of Posttranslational Modifications of p53
}

\author{
Qing-Duan Fan, ${ }^{1,2}$ Guang Wu, ${ }^{3,4}$ and Zeng-Rong Liu ${ }^{1}$ \\ ${ }^{1}$ Institute of Systems Biology, Shanghai University, 99 Shangda Road, Shanghai 200444, China \\ ${ }^{2}$ College of Fundamental Studies, Shanghai University of Engineering Science, 333 Longteng Road, Shanghai 201620, China \\ ${ }^{3}$ Guangxi Academy of Sciences, 98 Daling Road, Nanning, Guangxi 530007, China \\ ${ }^{4}$ DreamSciTech, Apartment 207, Zhencaili 26, Zhujiang Road, Hexi District, Tianjin 300222, China
}

Correspondence should be addressed to Qing-Duan Fan; fanqingduan@163.com

Received 19 February 2014; Accepted 6 April 2014; Published 12 May 2014

Academic Editor: Lei Chen

Copyright (C) 2014 Qing-Duan Fan et al. This is an open access article distributed under the Creative Commons Attribution License, which permits unrestricted use, distribution, and reproduction in any medium, provided the original work is properly cited.

The latest experimental evidence indicates that acetylation of p53 at K164 (lysine 164) and K120 may induce directly cell apoptosis under severe DNA damage. However, previous cell apoptosis models only studied the effects of active and/or inactive p53, that is, phosphorylation/dephosphorylation of p53. In the present paper, based partly on Geva-Zatorsky et al. (2006) and Batchelor et al. (2008), we propose a new cell apoptosis network, in which p53 has three statuses, that is, unphosphorylated p53, phosphorylated p53, and acetylated p53. The time delay differential equations (DDEs) are formulated based on our network to investigate the dynamical insights of p53-induced cell apoptosis. In agreement with experiments (Loewer et al. (2010)), our simulations indicate that acetylated p53 accumulates gradually and then induces the proapoptotic protein Bax under enough DNA damage. Moreover, phosphorylated p53 oscillates and initiates cell repair during DNA damage.

\section{Introduction}

The tumor suppressor p53, a multifunctional transcription factor, plays an essential role in regulating cellular processes including cell cycle arrest and apoptosis [1]. The importance of $\mathrm{p} 53$ also lies in its mutation in over $50 \%$ of human cancers. There are three main routes, namely, DNA double-strand break (DSB), ultraviolet light (UV), and oncogenes, which can result in an increase in p53 expression. Experiments demonstrate that cell outcomes depend on the extent of DNA damage, which probably decides the number of $\mathrm{p} 53$ pulses or p53 oscillation $[2,3]$. Experimentally, p53 regulates cell cycle protein p21, PUMA, BCL-2, PTEN, Bax, Bak, and so on [4], and a cell survives when DNA damage is reparable or commits suicide when irreparable. That is to say, a cell has these two means of avoiding cancer under DNA damage [5]. It follows that the mechanism of repair or apoptosis is closely linked with $\mathrm{p} 53$.

There are large quantities of experimental and theoretical researches on p53 networks, in which hundreds of genes and their corresponding proteins are involved. Consequently, the networks include many positive and negative feedback loops acting upon p53. The most prominent of them is the feedback loop between p53 and Mdm2 (mouse double minute 2), which has been considered in many dynamical models. p53 and Mdm2 show nondecaying oscillations in an individual cell, while demonstrating decaying oscillations in cell population, which may be ascribed to aggregate behavior of cells [1]. The ATM- (Ataxia Telan-giesctasia Mutated-) p53wipl (wild-type p53-induced phosphatase 1) feedback loop plays an important role in the generation of p53 pulses [6]. These intricate positive and negative feedback loops display various dynamical behaviors [7]. The reliable and flexible mechanism can avoid the premature apoptosis resulting from fluctuations in p53 levels. It is indicated that p53 is modified in a progressive manner and that $\mathrm{p} 53$ is divided into p53-arrest and $\mathrm{p} 53$-apoptosis in the integrative model [8]. Moreover, high constant levels of active p53 may trigger apoptosis quickly once the decision favoring death is made in seriously damaged cells. 
There are several "protein-protein" and "protein-mRNA" dynamical models which describe in detail intracellular signalling of the protein p53. Many theoretical results are obtained in determinate systems. Based on two compartments, nucleus and cytoplasm, an ODEs model is formulated to exhibit that the accumulation of p53 after triggering of ATM under DNA damage. The model also shows robustness of the protein oscillatory dynamics in response to different cellular environments [9]. A set of ordinary differential equations in single cell level shows p53 oscillations in each compartment, nucleus or cytoplasm, and between the two compartments [10]. Based on a sequence of precisely timed drug additions, the authors formulate a computational model, which shows that the dynamic of p53 changes from a pulse to a sustained response [11]. There are also quite a few stochastic systems, where the stochasticity of regulation on p53 shows high heterogeneity and stochastic character of single-cell response [12-15]. Up to now, only phosphorylation modification has been considered in dynamical models.

In fact, a cell is regulated accurately by many posttranslational modifications of $\mathrm{p} 53$, which can initiate a program of cell repair or apoptosis at different levels of DNA damage. Methylation of p53 facilitates its subsequent acetylation and protects $\mathrm{p} 53$ from ubiquitination [16]. Phosphorylation of p53 is important for inducing p21, a prime inhibitor of cell cycle. Recently, experiments find that a number of external and internal insults induce acetylation and accumulation of $\mathrm{p} 53$, via MYBBP1A, RPL5, and RPL11, without phosphorylation [17-19]. It is shown that acetylation of p53 at K164 and K120 may promote cell apoptosis rather than cell arrest $[20,21]$. It is observed that p53 may fundamentally switch from pulsing under slight damage to monotonic increase under severe damage [22]. However, there is no corresponding theoretical result about the dynamics of acetylation of p53 up to now.

In the paper, we distinguish functionally the effect of acetylation from phosphorylation of p53 and develop the DDEs of p53 transcriptional regulatory networks based on new experiments $[18,22]$ and related researches $[1,2,6,8,12]$. We pay special attention to the effect of acetylation of p53 and the proapoptotic protein Bax in the case of DNA damage. In agreement with experiments, our simulations indicate that acetylated p53 accumulates gradually with serious DNA damage and induces Bax when p53 surpasses a level.

\section{Methods and Models}

Methylation allows p53 to be inactive, in normal circumstances p53 is samilarly inactive, so we may regard the initial status of p53 to be inactive without consideration of methylation. Dynamical models of two statuses of p53, that is, inactive p53 and active p53/phosphorylated p53, have been studied extensively $[2,3,6,8]$. It is indicated that active forms of p53, such as phosphorylated p53 and acetylated p53, have different dynamics and functions experimentally [18]. Interestingly, p53 can be acetylated and accumulates without phosphorylation [17]. Acetylation of p53 on K120 is crucial to p53 dependent apoptosis but is dispensable for p53-mediated growth arrest [19]. In another experiment,

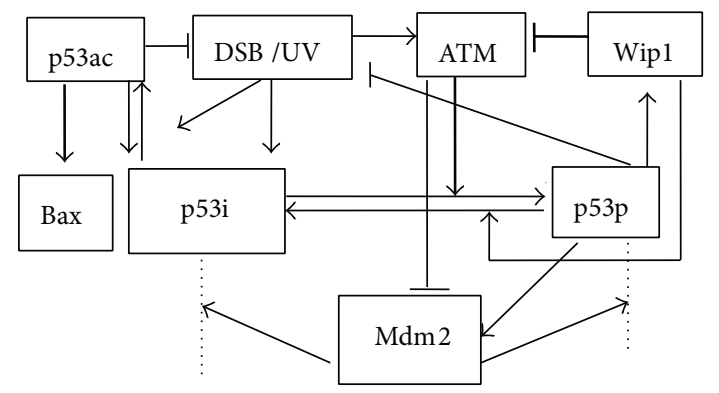

FIgURE 1: Schematic depiction of the model for the p53 networks in response to DNA damage. Transcription regulation is denoted in thick line. Degradation is denoted in dotted line. Sharp and blunt arrow denote activation and suppression, respectively. ATM's catalyzing phosphorylation of $\mathrm{Mdm} 2$, allowing $\mathrm{Mdm} 2$ easier to degrade, is regarded as repressive.

acetylation-defective of p53 at k120 can selectively block the transcription of proapoptotic target genes such as Bax and PUMA but has no obvious effect on cell cycle inhibitor p21 [16]. Accordingly, it is necessary that we explore the relation between the means of modifications and cell outcomes. It is shown that $\mathrm{p} 53$ may transcribe a few proapoptotic genes such as PUMA, Noxa, Bax, and Bid under excessive DNA damage. Moreover, Bax is very much related to cell apoptotic [23]. For simplicity, we choose Bax as a proapoptotic marker. Because both cell repair and cell apoptosis can lessen DSB; to put it in another way, p53ac and p53p can be regarded as inhibiting DSB. Based on preexisting researches $[2,3,6]$, we obtain the following modular schematic depiction in Figure 1.

Considering the tetramer of p53 as a transcription factor, p53-induced Bax is characterized by a Hill function. When phosphorylation and dephosphorylation of p53 are considered separately, a near-optimal switch is possible via Hill equation, where the Hill coefficient equals the number of phosphorylation sites [24]. It is shown that p53 can be phosphorylated at sites S15, T18, S20, S37, S378, and S392 [25]; but Hill coefficient usually ranges between 2 and 4 [24]. Here we denote the ATM-induced phosphorylation of p53 and wipl-induced dephosphorylation of p53 via Hill equation. For simplicity, Hill coefficients take 4. Specially, wipl-induced dephosphorylated of ATM is denoted by Hill equation, where Hill coefficient takes 2 for ATM protein is a dimer. Active p53 has a weaker interaction with $\mathrm{Mdm} 2$ than inactive p53 and hence a lower degradation rate [1].

Phosphorylated $\mathrm{p} 53$ has a lower degradation rate than inactive p53 because the binding of it with $\mathrm{Mdm} 2$ is weaker than inactive p53 [1]. Additionally, the acetylated residues cannot be ubiquitylated by Mdm2 [26]. In the model, two delays are considered because the transcription of $\mathrm{Mdm} 2$ by p53 needs time [2] and wip1 expression with a delay would allow p53-induced cell repair [1]. Sustained damage leads to acetylation and accumulation of p53 [17]; however, no acetylation was detected in response to a transient and lowlevel damage (Figure 6(d) in [18]). In other words, p53 is acetylated only when DNA damage surpasses a certain level, 
TABLE 1: The meaning and values of parameters in the systems (1)(8).

\begin{tabular}{|c|c|c|}
\hline Parameter & Biological meaning & Value \\
\hline$b_{p}$ & Inactive $\mathrm{p} 53$ production rate & 3 \\
\hline$b_{\mathrm{sp}}$ & $\begin{array}{l}\text { p53 saturating phosphorylate rate by } \\
\text { ATM/ATR }\end{array}$ & $10^{*} / 1$ \\
\hline$b_{m}$ & $\mathrm{p} 53$-dependent $\mathrm{Mdm} 2$ production rate & 0.9 \\
\hline$b_{\mathrm{mi}}$ & p53-independent $\mathrm{Mdm} 2$ production rate & $0.2^{*}$ \\
\hline$b_{i}$ & Inhibitor Wip1 production rate & $0.25^{*}$ \\
\hline$b_{s}$ & Signal ATM production rate & $10^{*}$ \\
\hline$b_{\mathrm{pac}}$ & $\begin{array}{l}\text { Acetylation speed of p } 53 \text { under DSB/UV } \\
\text { damage }\end{array}$ & $0.1 / 0.02$ \\
\hline$b_{\mathrm{Bax}}$ & Saturating production rate of $\mathrm{Bax}$ & 0.04 \\
\hline$a_{\mathrm{mpi}}$ & $\begin{array}{l}\text { Mdm2-dependent p53 inactive degradation } \\
\text { rate }\end{array}$ & $5^{*}$ \\
\hline$a_{\mathrm{pi}}$ & Inactive p53 degradation rate & $2^{*}$ \\
\hline$a_{\mathrm{mpa}}$ & $\begin{array}{l}\text { Mdm2-dependent active p53 degradation } \\
\text { rate }\end{array}$ & 0.35 \\
\hline$a_{\mathrm{sm}}$ & Signal-dependent $\mathrm{Mdm} 2$ inactivation rate & 0.5 \\
\hline$a_{\text {wpa }}$ & $\begin{array}{l}\text { Wipl-dependent dephosphorylation rate of } \\
\text { p53 }\end{array}$ & 2.8 \\
\hline$a_{m}$ & Mdm2 degradation rate & $1^{*}$ \\
\hline$a_{i}$ & Inhibitor Wip1 degradation rate & $0.7^{*}$ \\
\hline$a_{i s}$ & Wipl-dependent Signal degradation rate & $50^{*}$ \\
\hline$a_{s}$ & ATM degradation rate & $7.5^{*}$ \\
\hline$a_{\mathrm{pac}}$ & acetylation of p53 degradation rate & 0.05 \\
\hline$a_{\text {depac }}$ & deacetylation rate of $\mathrm{p} 53$ & 0.05 \\
\hline$a_{\mathrm{Bax}}$ & Bax degradation rate & 0.04 \\
\hline$\tau_{1}$ & Time delay of Mdm 2 transcription by $\mathrm{p} 53$ & $0.7^{*}$ \\
\hline$\tau_{2}$ & Time delay of Wip1 transcription by p53 & $1.25^{*}$ \\
\hline ta & $\begin{array}{l}\text { Concentration of Bax for half maximal } \\
\text { p53ac production }\end{array}$ & 0.15 \\
\hline ts & $\begin{array}{l}\text { Concentration of ATM for half-maximal p53 } \\
\text { production }\end{array}$ & $1^{*}$ \\
\hline$t i$ & $\begin{array}{l}\text { Concentration of wip1 for half-maximal } \\
\text { Signal degradation }\end{array}$ & $0.2^{*}$ \\
\hline$\theta_{0}$ & The threshold of DSBs for acetylating p53 & 0.1 \\
\hline$k_{\text {rep }}$ & Repair rate of DSBs & 0.1 \\
\hline$n_{1}$ & $\begin{array}{l}\text { Hill coefficients for phosphorylation and } \\
\text { dephosphorylate of p53 }\end{array}$ & 4 \\
\hline$n_{2}$ & $\begin{array}{l}\text { Hill coefficients for dephosphorylation of } \\
\text { ATM by wip }\end{array}$ & 2 \\
\hline$[\mathrm{p} 53 \mathrm{i}]_{0}$ & The initial condition of inactive p53 & 1 \\
\hline$[\mathrm{p} 53 \mathrm{p}]_{0}$ & The initial condition of phosphorylated p53 & 0 \\
\hline$[\mathrm{Mdm} 2]_{0}$ & The initial condition of $\mathrm{Mdm} 2$ & 0.2 \\
\hline$[\text { wipl }]_{0}$ & The initial condition of wipl & 0 \\
\hline$[\mathrm{ATM}]_{0}$ & The initial condition of ATM & 0 \\
\hline$[\mathrm{p} 53 \mathrm{ac}]_{0}$ & The initial condition of acetylated p53 & 0 \\
\hline$[\mathrm{Bax}]_{0}$ & The initial condition of $\mathrm{Bax}$ & 0 \\
\hline$[\mathrm{DSBs}]_{0}$ & The initial conditions of two type of DSBs & $3 / 0.3^{* *}$ \\
\hline
\end{tabular}

${ }^{*}$ denotes parameters from $[2,6]$, the others are estimated. ${ }^{* *}$ denotes serious/slight DSBs.

$\theta_{0}$. It is shown that the transcription rate of $\mathrm{p} 53$ is independent of DNA damage; moreover, there exists an increase in translation rate of $\mathrm{p} 53$ following gamma irradiation, so signal strength, ATM, can be denoted by $\theta(x)$. Here $\theta$ (damage) equals 1 if damage exists, zero otherwise [2]. According to Figure 1 and the dynamical model in $[2,3,6]$, we further formulate a set of DDEs:

$$
\begin{aligned}
\frac{d \mathrm{p} 53 \mathrm{i}}{d t}= & b_{p}-a_{\mathrm{mpi}} \mathrm{Mdm} 2 \cdot \mathrm{p} 53 \mathrm{i}-b_{\mathrm{sp}} \mathrm{ATM} \frac{\mathrm{p} 53 \mathrm{i}^{n 1}}{t s^{n}+\mathrm{p}^{n} 5 \mathrm{i}^{n 1}} \\
& -a_{\mathrm{pi}} \mathrm{p} 53 \mathrm{i}+a_{\mathrm{wpa}} \operatorname{wip} 1 \frac{\mathrm{p} 53 \mathrm{p}^{n 1}}{t i^{n 1}+\mathrm{p} 53 \mathrm{p}^{n 1}} \\
& -b_{\mathrm{pac}} \mathrm{p} 53 \mathrm{i} \max \left(\mathrm{DSB}-\theta_{0}, 0\right)+a_{\mathrm{depac}} \mathrm{p} 53 \mathrm{ac}
\end{aligned}
$$

$$
\begin{aligned}
\frac{d \mathrm{p} 53 \mathrm{p}}{d t}= & b_{\mathrm{sp}} \operatorname{ATM} \frac{\mathrm{p} 53 \mathrm{i}^{n 1}}{t s^{n 1}+\mathrm{P} 53 \mathrm{i}^{n 1}}-a_{\mathrm{mpa}} \operatorname{Mdm} 2 \cdot \mathrm{p} 53 \mathrm{p} \\
& -a_{\mathrm{wpa}} \text { wip } 1 \frac{\mathrm{p} 53 \mathrm{p}^{n 1}}{t i^{n 1}+\mathrm{p} 53 \mathrm{p}^{n 1}},
\end{aligned}
$$

$$
\frac{d \mathrm{ATM}}{d t}=b_{s} \theta(\mathrm{DSB})-a_{\mathrm{is}} \operatorname{wip} 1 \frac{\mathrm{ATM}^{n 2}}{t i^{n 2}+\mathrm{ATM}^{n 2}}-a_{s} \mathrm{ATM}
$$

$$
\begin{aligned}
\frac{\mathrm{dp} 53 \mathrm{ac}}{d t}= & b_{\mathrm{pac}} \mathrm{p} 53 \mathrm{i} \max \left(\mathrm{DSB}-\theta_{0}, 0\right) \\
& -a_{\mathrm{pac}} \mathrm{p} 53 \mathrm{ac}-a_{\mathrm{depac}} \mathrm{p} 53 \mathrm{ac},
\end{aligned}
$$

$$
\frac{d \mathrm{Bax}}{d t}=b_{\mathrm{Bax}} \frac{\mathrm{p} 53 \mathrm{ac}^{n 1}}{t a^{n 1}+\mathrm{p} 53 \mathrm{ac}}-a_{\mathrm{Bax}} \mathrm{Bax},
$$$$
\frac{d \mathrm{DSB}}{d t}=-k_{\mathrm{rep}} \mathrm{DSB}(\mathrm{p} 53 \mathrm{p}+\mathrm{p} 53 \mathrm{ac}),
$$

where p53i, p53p, and p53ac represent inactive p53, phosphorylated p53, and acetylated p53, respectively. In (1), the first term represents p53i synthesis; the second one, catalytic degradation of $\mathrm{Mdm} 2$; and the third one, phosphorylation; the fourth to the last one describe self-degradation, dephosphorylation, and acetylation, respectively. In (2), the first term denotes phosphorylation of p53 by ATM; the second one, ubiquitination by $\mathrm{Mdm} 2$; and the third one, wip-dependent dephosphorylation. In (3), the first term represents Mdm2 synthesis speed; the second one, activating $\mathrm{Mdm} 2$ via $\mathrm{p} 53 \mathrm{p}$, where the delay, $\tau_{1}$, denotes the time for the transport of p53 from cytoplasm to nucleus and the transcription of Mdm2; the third one, catalyzation of ATM; and the last one, self-degradation. In (4), the first term represents activating wipl via p53, where the delay, $\tau_{2}$, is introduced owing to the time for the transport of p53 and the transcription of wip1 by $\mathrm{p} 53$ and the second one, self-degradation. In (5), the first term represents exciting ATM induced by DSB; the second one, dephosphorylation by wipl; and the third 
one, self-degradation. In (6), the first term represents p53 acetylation caused by DSB, wherein $\max (x)$ refers to the maximum function and the second one, self-degradation of p53ac. In (7), the first term represents Bax induced by p53 and the second one, self-degradation of Bax. (8) denotes the reduction rate of DSB owning to cell repair and apoptosis. For simplicity, all the parameters in the system consisting of (1)(8) are listed in Table 1.

\section{Results}

Various exogenous or endogenous stimuli can generate damaged DNA. DSBs and UV are the main types of stimuli, which can activate p53 and subsequently command cell outcomes. DSBs are discussed in two cases, that is, pulsing and repairable DSBs, so we simulate the model according to three cases of DNA damage.

Firstly, we consider the system equations ((1)-(7)) with DSBs at pulsing level. When $10<t<40$, DSBs take 3 , otherwise DSBs take $0 . \theta_{0}$, the threshold of DSB for acetylating p53, takes arbitrarily 0.1 . The initial conditions of p53i and Mdm2 take 1 and 0.2, respectively; the others take 0. The numerical simulations with Matlab 7.10 (Mathworks) are shown in Figure 2(a), which indicates that p53p has several oscillations with a constant period of about $6 \mathrm{hs}$ and that p53ac accumulates. When p53ac surpasses a level, 0.2 or so, it activates proapoptosis protein Bax. From a biological standpoint, it is reasonable that Bax is activated when p53ac reaches a sufficient level. It is better that the threshold can be testified by experiments. In our simulations, Bax is up to 0.6, which is a rather high level, and we think that cell apoptosis should occur. Experimentally, DSBs are basal in proliferating cell, but cell apoptosis does not occur. p53ac hardly expresses when DSBs are smaller than 0.3, and Bax is also the case (simulations are shown in Figure 2(b)).

Secondly, DSBs decrease under cell pair or cell apoptosis. We consider the system equations (1)-(8) with the parameters and initial conditions set in Table 1 and get numerical simulation shown in Figures 2(c) and 2(d). It is shown that p53p oscillates owing to decreasing DSBs, with a constant period similar to that in the system equations (1)-(7), while p53ac accumulates under serious DNA damage. p53ac ascends and then descends with the decrease of DSBs. When p53ac surpasses a certain level, 0.2 or so, it induces Bax. These simulation results, such as a sufficient level of p53ac activating Bax, are consistent with the biological fact that activation of some proteins need enough inducer. With parameters set in Table 1, simulations show that in the system p53p probably has 5 pulses when $[\mathrm{DSBs}]_{0}$ is 3 (Figure $2(\mathrm{c})$ ). When [DSBs $]_{0}$ drops to 0.3 , p53ac and Bax are hardly expressed, and p53p has 2 pulses (Figure $2(\mathrm{~d})$ ). In the cases that $[\mathrm{DSBs}]_{0}$ is 1 or 20 , cell has only 4 pulses, but cells have different outcomes, that is, cell repair and cell apoptosis (simulations not shown). The smaller $[\mathrm{DSBs}]_{0}$ is, the smaller p53ac and the number of pulse of $\mathrm{p} 53 \mathrm{p}$ are. On the contrary, the greater $[\mathrm{DSBs}]_{0}$ is, the faster p53ac accumulates but the smaller the number of pulse of $\mathrm{p} 53 \mathrm{p}$ is, which appear to show that cell apoptosis is faster.
Now we consider the robustness of the system parameters. Simulations of the system shown in Figures 2(c) and 2(d) with new parameters perturbation and with cited parameters are shown in Figure 3 . When $b_{\text {pac }}, b_{\mathrm{Bax}}$, and $a_{\mathrm{depac}}$ increase by $10 \%$, and $a_{\mathrm{pac}}$ and $a_{\mathrm{Bax}}$ decrease by $10 \%$ in parameters of Figure 2(c), simulations of the p53 networks are shown in Figure $3(\mathrm{a})$. When $b_{\mathrm{pac}}, b_{\mathrm{Bax}}$, and $a_{\mathrm{depac}}$ decrease by $10 \%$ and $a_{\mathrm{pac}}, a_{\mathrm{Bax}}$, and $k_{\text {rep }}$ increase by $10 \%$ in parameters of Figure 2(d), simulations are shown in Figure 3(b). Our new parameters increase or decrease by $10 \%$, qualitative characteristics change little except for the position of the equilibrium point (Figure not shown). It is evident that steady points of p53i and Mdm2 do not change much comparing to that of using the initial parameters values. However, p53p and Mdm2 still oscillate while p53ac and Bax accumulate.

At last, when DNA is damaged by ultraviolet rays (UV), ATR, instead of ATM, phosphorylates p53 and Mdm2, except that dephosphorylation of wipl on ATR is dispensable, the other pathways do not change. Consequently, we have the following differential equations in response to UV:

$$
\begin{aligned}
& \frac{d \mathrm{p} 53 \mathrm{i}}{d t}=b_{p}-a_{\mathrm{mpi}} \mathrm{Mdm} 2 \mathrm{p} 53 \mathrm{i}-b_{\mathrm{sp}} \operatorname{ATR} \frac{\mathrm{p} 53 \mathrm{i}^{n 1}}{t s^{n 1}+\mathrm{p} 53 \mathrm{i}^{n 1}} \\
& -a_{\mathrm{pi}} \mathrm{p} 53 \mathrm{i}+a_{\mathrm{wpa}} \text { wip } 1 \frac{\mathrm{p} 53 \mathrm{p}^{n 1}}{t i^{n 1}+\mathrm{p} 53 \mathrm{p}^{n 1}} \\
& -b_{\text {pac }} \text { p53i } \theta\left(\mathrm{UV}-\theta_{0}\right) \text {, } \\
& \frac{d \mathrm{p} 53 \mathrm{p}}{d t}=b_{\mathrm{sp}} \operatorname{ATR} \frac{\mathrm{p} 53 \mathrm{i}^{n 1}}{t s^{n 1}+\mathrm{p} 53 \mathrm{i}^{n 1}}-a_{\mathrm{mpa}} \operatorname{Mdm} 2 \\
& \cdot \mathrm{p} 53 \mathrm{p}-a_{\mathrm{wpa}} \text { wip } 1 \frac{\mathrm{p} 53 \mathrm{p}^{n 1}}{t i^{n 1}+\mathrm{p} 53 \mathrm{p}^{n 1}} \\
& \frac{d \mathrm{Mdm} 2}{d t}=b_{m} \mathrm{p} 53 \mathrm{p}\left(t-\tau_{1}\right)+b_{\mathrm{mi}} \\
& -a_{\mathrm{sm}} \text { ATR Mdm2 }-a_{m} \mathrm{Mdm} 2, \\
& \frac{d \mathrm{ATR}}{d t}=b_{s} \mathrm{UV}-a_{s} \mathrm{ATR}, \\
& \frac{\mathrm{dp} 53 \mathrm{ac}}{d t}=b_{\mathrm{pac}} \mathrm{p} 53 \mathrm{i} \theta\left(\mathrm{UV}-\theta_{0}\right) \\
& -a_{\mathrm{pac}} \mathrm{p} 53 \mathrm{ac}-a_{\mathrm{depac}} \mathrm{p} 53 \mathrm{ac},
\end{aligned}
$$

where (9)-(13) are based on [6].

We regard damage capability of UV as one-tenth of DSB. Paramters $b_{\mathrm{pac}}, a_{\mathrm{pac}}, b_{\mathrm{Bax}}, a_{\mathrm{Bax}}, \theta_{0}$ and the initial conditions are set and shown in Table 1 . Take UV $=8$ for $0 \leq t<15$, and take 0 for $t \geq 15$ as [6], the simulations of (4), (7), and (9)-(13) are shown in Figure 4. It is indicated that during slight DNA damage, p53p exhibits a small pulse so as to induce cell cycle arrest/cell repair, and then returns to the initial condition. It is worth noting that $\mathrm{p} 53 \mathrm{ac}$ and cell apoptosis are little when $\mathrm{UV} \leq 10$, which is consistent with experimental results [6]. Total p53 has a pulse as phosphorylated p53 does and then returns to the basal level. 


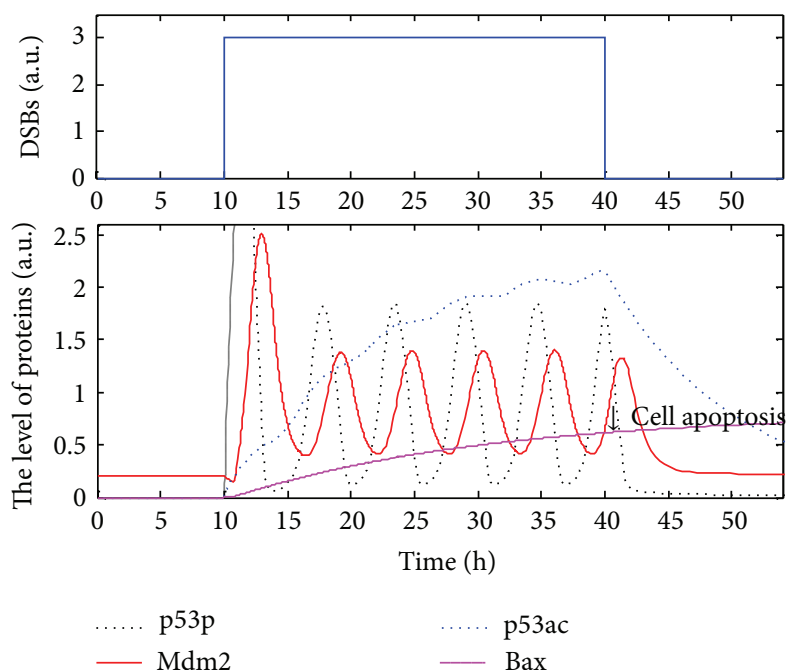

(a)

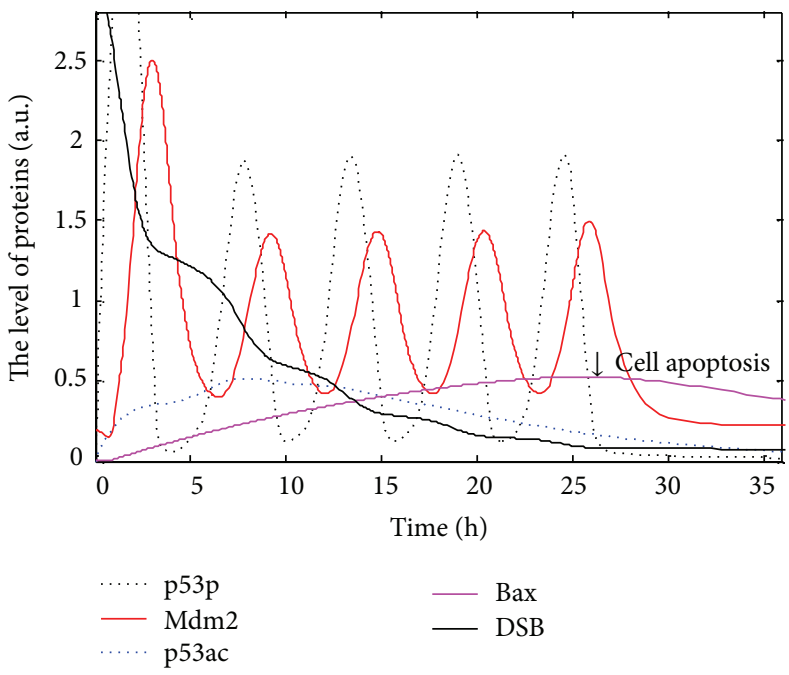

(c)
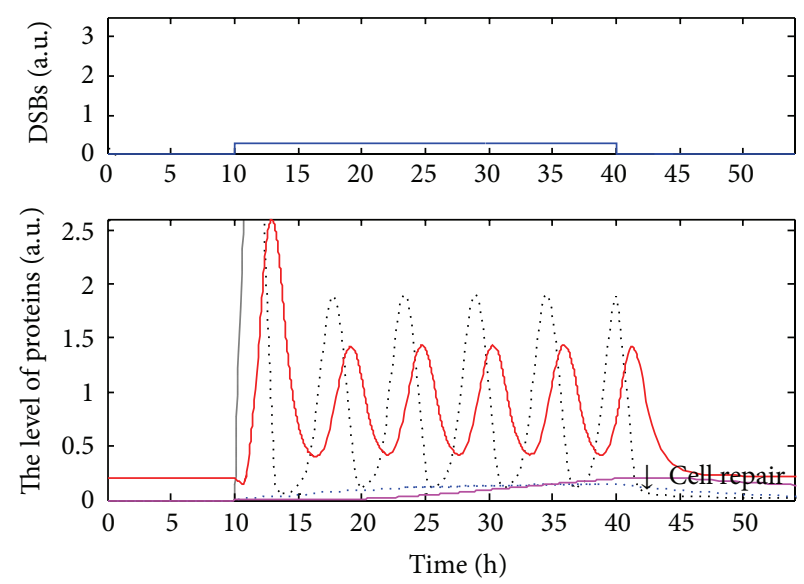

..... $\mathrm{p} 53 \mathrm{p}$

— $\mathrm{Mdm} 2$

…. p53ac

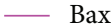

(b)



(d)

FIGURE 2: Simulations of the p53 regulatory networks under different types of DSBs. Black dotted lines indicates concentration of p53p; red solid lines Mdm2; blue dotted lines p53ac; carmine solid lines Bax; and black solid line DSB. (a, b) Pulsating DSB. (a) Pulse of DSBs takes 3 (a) and 0.3 (b). (c, d) DNA repair via p53 is considered. The initial DSBs take 3 (c) and 0.3 (d). (a, c) p53p oscillates while p53ac accumulates and induces cell apoptosis. (b, d) p53p oscillates while p53ac is hardly expressed during cell repair.

\section{Conclusion}

The tumor suppressor p53, a most frequently mutated protein in cancer cells, is a key regulator in cell cycle. The latest experiments $[17,18,24]$ show that posttranslational modifications of p53, such as phosphorylation and acetylation, are closely linked with cell repair and cell apoptosis. In order to interpret the experimental phenomenon, we develop the regulatory networks and the DDEs model and discuss the dynamics of modifications of p53. Experimentally, acetylation of p53 at $\mathrm{K} 120$ and K164 plays an important role in regulating proapoptotic protein [16-19]. It is indicated exactly that p90 is critical to p53-mediated cell apoptosis through promoting acetylation of p53; moreover, p90 has no obvious effects on p53-mediated cell cycle arrest but it is specifically needed for p53-mediated apoptosis [27]. The phenomena that p53ac accumulates and activates proapoptotic protein Bax only under serious DNA damage (Figures 2(a) and 2(c)) and that the pulses of $\mathrm{p} 53 \mathrm{p}$ and a little $\mathrm{p} 53 \mathrm{ac}$ allow cell to reenter cell cycle under slight DNA damage (Figures 2(b) and 2(d)) are consistent with the latest experiments $[2,6,18]$. The robustness analysis of the model (Figure 3 ) shows that the accumulation of $\mathrm{p} 53 \mathrm{ac}$ and the number of pulse of $\mathrm{p} 53 \mathrm{p}$ depend on the extent of DNA damage. The number of pulses of p53p, which means cell repair, should lie on cell repair or cell apoptosis under different levels of DNA damage [28]. UV can lead to single strand break, a kind of slight DNA damage, which usually activates $\mathrm{p} 53 \mathrm{p}$ and allows cell to reenter cell cycle (Figure 4). Accordingly, posttranslational modifications of p53 can help us know when and why a cell 


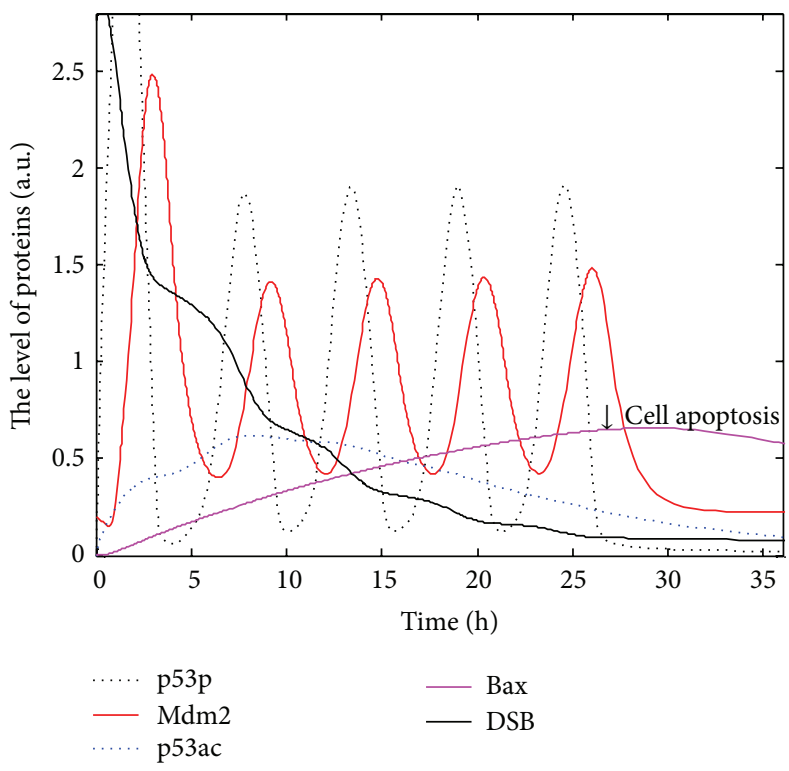

(a)

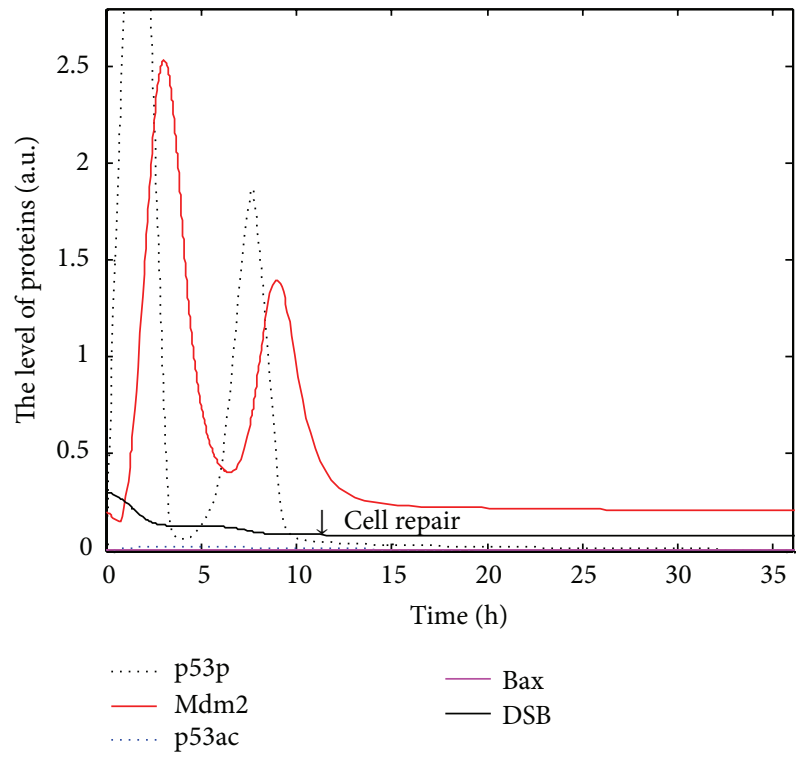

(b)

Figure 3: Parameters robustness of the p53 regulatory networks. Black dotted line indicates concentration of p53p; red solid line Mdm2; blue dotted line p53ac; carmine solid line Bax; and black solid line DSB. (a) Production rates decrease 10\% and degradation rates increase $10 \%$ in Figure 2(c). (b) Production rates increase $10 \%$ and degradation rates decrease $10 \%$ in Figure 2(d).

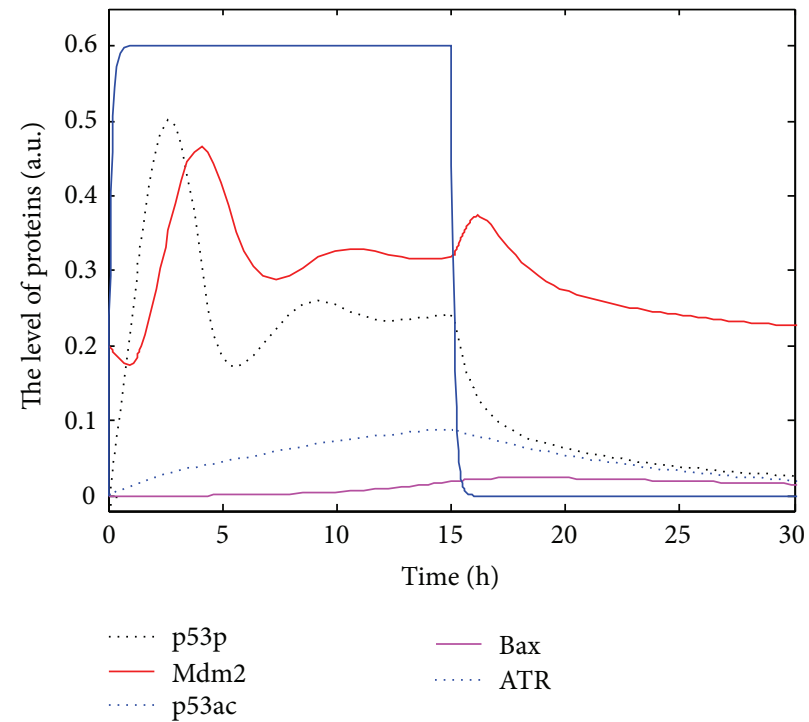

FIGURE 4: The dynamics of all kinds of p53 networks in response to UV $=8$. Black dotted line indicates concentration of p53p; red solid line Mdm2; blue dotted line p53ac; carmine solid line Bax; and blue solid line ATR.

selects programmed cell death or cell repair. In other words, posttranslational modifications of $\mathrm{p} 53$ will be beneficial to the treatment of tumors as an innovative therapeutic strategy.

Since cell kinetics is complicated and precisely accurate, it is very difficult to consider all details of posttranslational modifications of p53 [25, 27]. Reference [29] shows phosphorylation may allow p53 stabilization, enhancement of DNAbinding, and activation of its cell-cycle arrest pathway, while acetylation may allow p53 to activate its apoptotic pathway. Yet there are exceptions. For example, phosphorylation at S46 is critical to the induction of proapoptotic genes p53AIP1 (p53-regulated Apoptosis-Inducing Protein 1), but it is not required for the induction of cell cycle inhibitor p21. Phosphorylation and acetylation of p53 probably have synergistic effects on cell cycle, such as acetylation of p53 at k320 may also induce p21 and repress apoptosis [30]. Methylation may allow p53 to be a transcriptionally inactive state; however, methylation of p53 facilitates its subsequent acetylation and protects p53 from ubiquitination [16]. Neddylation and sumoylation have not been demonstrated to affect p53 stability yet. 
Additionally, p53 may regulate the transcription expression of many miRNAs, such as MiR-34 and MiR-200. On the other hand, the expression and modification of $\mathrm{p} 53$ are also regulated by quantities of MiRNAs [31, 32]. Accordingly, the mechanism of posttranslational modification needs exploration for a long time both in experiment and in theory, such as the mechanism of randomness of protein expression. Our further work will focus on more accurate posttranslational modifications of p53 in cell cycle.

\section{Conflict of Interests}

The authors declare that there is no conflict of interests regarding the publication of this paper.

\section{Acknowledgments}

The authors acknowledge support Grants from National Natural Science Foundation of China (Grants no. 10832006, 11172158, and 11101265).

\section{References}

[1] N. Geva-Zatorsky, N. Rosenfeld, S. Itzkovitz et al., "Oscillations and variability in the p53 system," Molecular Systems Biology, vol. 2, no. 1, 2006.

[2] E. Batchelor, C. S. Mock, I. Bhan, A. Loewer, and G. Lahav, "Recurrent initiation: a mechanismfor triggering p53 pulses in response to DNA damage," Molecular Cell, vol. 30, no. 3, pp. 277-289, 2008.

[3] L. Ma, J. Wagner, J. J. Rice, W. Hu, A. J. Levine, and G. A. Stolovitzky, "A plausible model for the digital response of p53 to DNA damage," Proceedings of the National Academy of Sciences of the United States of America, vol. 102, no. 40, pp. 14266-14271, 2005.

[4] C. Charvet, M. Wissler, P. Brauns-Schubert et al., "Phosphorylation of Tip60 by GSK-3 Determines the Induction of PUMA and Apoptosis by p53," Molecular Cell, vol. 42, no. 5, pp. 584596, 2011.

[5] H. C. Reinhardt and B. Schumacher, "The p53 network: cellular and systemic DNA damage responses in aging and cancer," Trends in Genetics, vol. 28, no. 3, pp. 128-136, 2012.

[6] E. Batchelor, A. Loewer, C. Mock, and G. Lahav, "Stimulusdependent dynamics of p53 in single cells," Molecular Systems Biology, vol. 7, article 488, 2011.

[7] S. L. Harris and A. J. Levine, "The p53 pathway: positive and negative feedback loops," Oncogene, vol. 24, no. 17, pp. 28992908, 2005.

[8] X.-P. Zhang, F. Liu, and W. Wang, "Two-phase dynamics of p53 in the DNA damage response," Proceedings of the National Academy of Sciences of the United States of America, vol. 108, no. 22, pp. 8990-8995, 2011.

[9] L. Dimitrio, J. Clairambault, and R. Natalini, "A spatial physiological model for p53 intracellular dynamics," Journal of Theoretical Biology, vol. 316, pp. 9-24, 2013.

[10] J. Eliaš, L. Dimitrio, J. Clairam-bault, and R. Natalini, “The p53 protein and its molecular network: modelling a missing link between DNA damage and cell fate," Biochimica et Biophysica Acta, vol. 1844, no. 1, Part B, pp. 232-247, 2014.
[11] J. E. Purvis, K. W. Karhohs, C. Mock et al., "p53 dynamics control cell fate," Science, vol. 336, no. 6087, pp. 1440-1444, 2012.

[12] T. Sun, W. Yang, J. Liu, and P. Shen, "Modeling the basal dynamics of P53 system," PLoS ONE, vol. 6, no. 11, Article ID e27882, 2011.

[13] B. Liu, S. Yan, and Q. Wang, "Intrinsic noise and Hill dynamics in the p53 system," Journal of Theoretical Biology, vol. 269, no. 1, pp. 104-108, 2011.

[14] K. Puszyński, B. Hat, and T. Lipniacki, "Oscillations and bistability in the stochastic model of p53 regulation," Journal of Theoretical Biology, vol. 254, no. 2, pp. 452-465, 2008.

[15] Y.-C. Hung and C.-K. Hu, "Constructive role of noise in p53 regulatory network," Computer Physics Communications, vol. 182, no. 1, pp. 249-250, 2011.

[16] G. S. Ivanov, T. Ivanova, J. Kurash et al., "Methylationacetylation interplay activates 553 in response to DNA damage," Molecular and Cellular Biology, vol. 27, no. 19, pp. 6756-6769, 2007.

[17] T. Kuroda, A. Murayama, N. Katagiri et al., "RNA content in the nucleolus alters p53 acetylation via MYBBP1A," EMBO Journal, vol. 30, no. 6, pp. 1054-1066, 2011.

[18] A. Loewer, E. Batchelor, G. Gaglia, and G. Lahav, "Basal dynamics of 553 reveal transcriptionally attenuated pulses in cycling cells," Cell, vol. 142, no. 1, pp. 89-100, 2010.

[19] Y. Tang, W. Zhao, Y. Chen, Y. Zhao, and W. Gu, "Acetylation is indispensable for p53 activation," Cell, vol. 133, no. 4, pp. 612626, 2008.

[20] S. M. Sykes, T. J. Stanek, A. Frank, M. E. Murphy, and S. B. McMahon, "Acetylation of the DNA binding domain regulates transcription-independent apoptosis by p53," Journal of Biological Chemistry, vol. 284, no. 30, pp. 20197-20205, 2009.

[21] H. M. Collins, M. K. Abdelghany, M. Messmer et al., "Differential effects of garcinol and curcumin on histone and p53 modifications in tumor cells," BMC Cancer, vol. 13, no. 1, article 37, 2013.

[22] X. Chen, J. Chen, S. Gan et al., "DNA damage strength modulates a bimodal switch of p53 dynamics for cell-fate control," BMC Biology, vol. 11, article 73, 2013.

[23] J. K. Sax, P. Fei, M. E. Murphy, E. Bernhard, S. J. Korsmeyer, and W. S. El-Deiry, "BID regulation by p53 contributes to chemosensitivity," Nature Cell Biology, vol. 4, no. 11, pp. 842849, 2002.

[24] X. Liu, L. Bardwell, and Q. Nie, "A combination of multisite phosphorylation and substrate sequestration produces switchlike responses," Biophysical Journal, vol. 98, no. 8, pp. 1396-1407, 2010.

[25] J.-P. Kruse and W. Gu, "Modes of p53 regulation," Cell, vol. 137, no. 4, pp. 609-622, 2009.

[26] D. W. Meek and C. W. Anderson, "Posttranslational modification of p53: cooperative integrators of function," Cold Spring Harbor Perspectives in Biology, vol. 1, no. 6, Article ID a000950, 2009.

[27] C. Dai, Y. Tang, S. Y. Jung, J. Qin, S. A. Aaronson, and W. $\mathrm{Gu}$, "Differential effects on p53-mediated cell cycle arrest vs. apoptosis by p90," Proceedings of the National Academy of Sciences of the United States of America, vol. 108, no. 47, pp. 18937-18942, 2011.

[28] X.-P. Zhang, F. Liu, Z. Cheng, and W. Wang, "Cell fate decision mediated by p53 pulses," Proceedings of the National Academy of Sciences of the United States of America, vol. 106, no. 30, pp. 12245-12250, 2009. 
[29] Z. Jiang, R. Kamath, S. Jin, M. Balasubramani, T. K. Pandita, and B. Rajasekaran, "Tip60-mediated acetylation activates transcription independent apoptotic activity of Abl," Molecular Cancer, vol. 10, article 88, 2011.

[30] C. D. Knights, J. Catania, S. Di Giovanni et al., "Distinct p53 acetylation cassettes differentially influence gene-expression patterns and cell fate," Journal of Cell Biology, vol. 173, no. 4, pp. 533-544, 2006.

[31] Z. Feng, C. Zhang, R. Wu, and W. Hu, "Tumor suppressor p53 meets microRNAs," Journal of Molecular Cell Biology, vol. 3, no. 1, pp. 44-50, 2011.

[32] H. Hermeking, "MicroRNAs in the p53 network: micromanagement of tumour suppression," Nature Reviews Cancer, vol. 12, no. 9, pp. 613-626, 2012. 


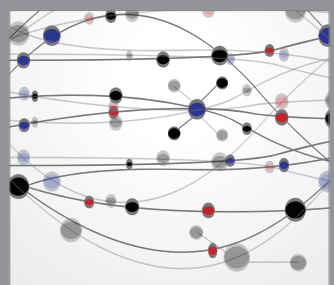

The Scientific World Journal
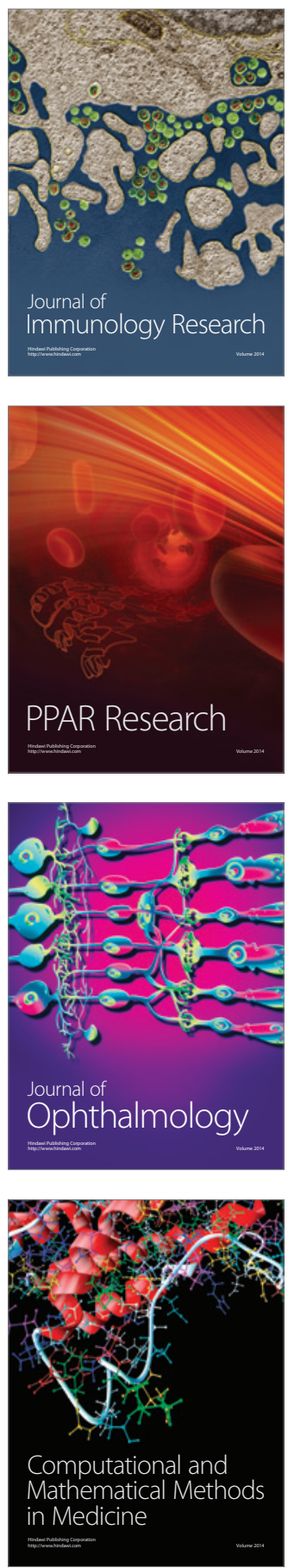

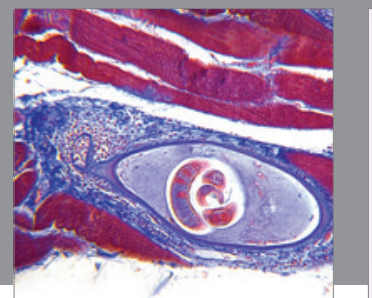

Gastroenterology

Research and Practice
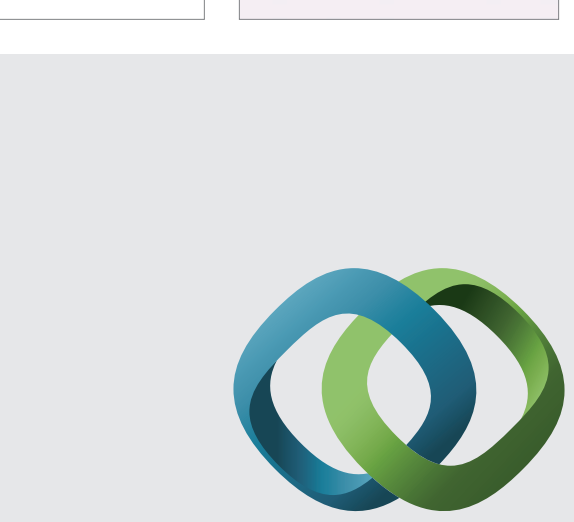

\section{Hindawi}

Submit your manuscripts at

http://www.hindawi.com
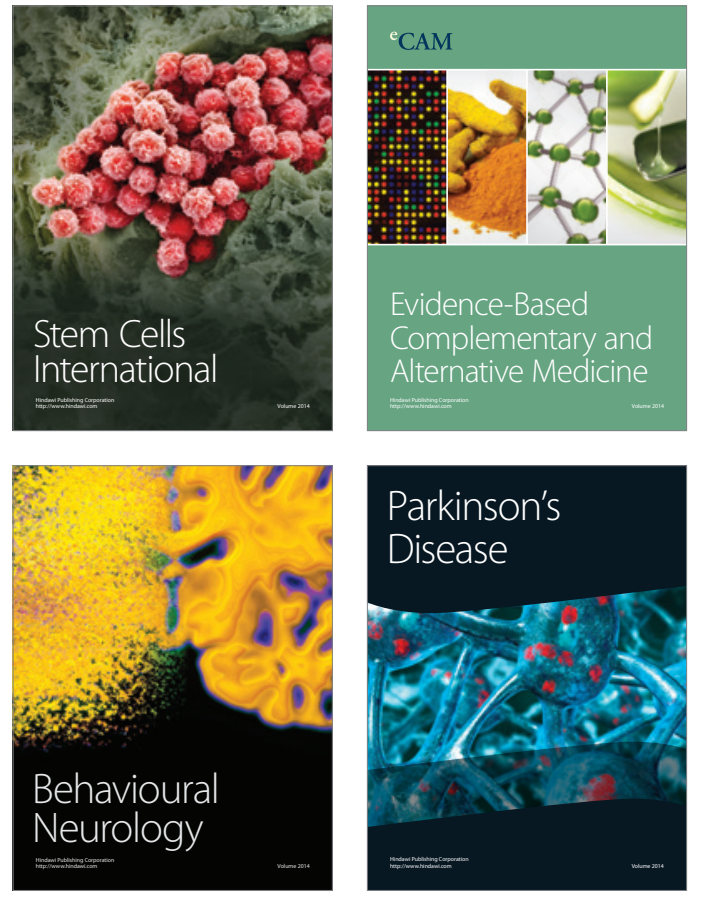
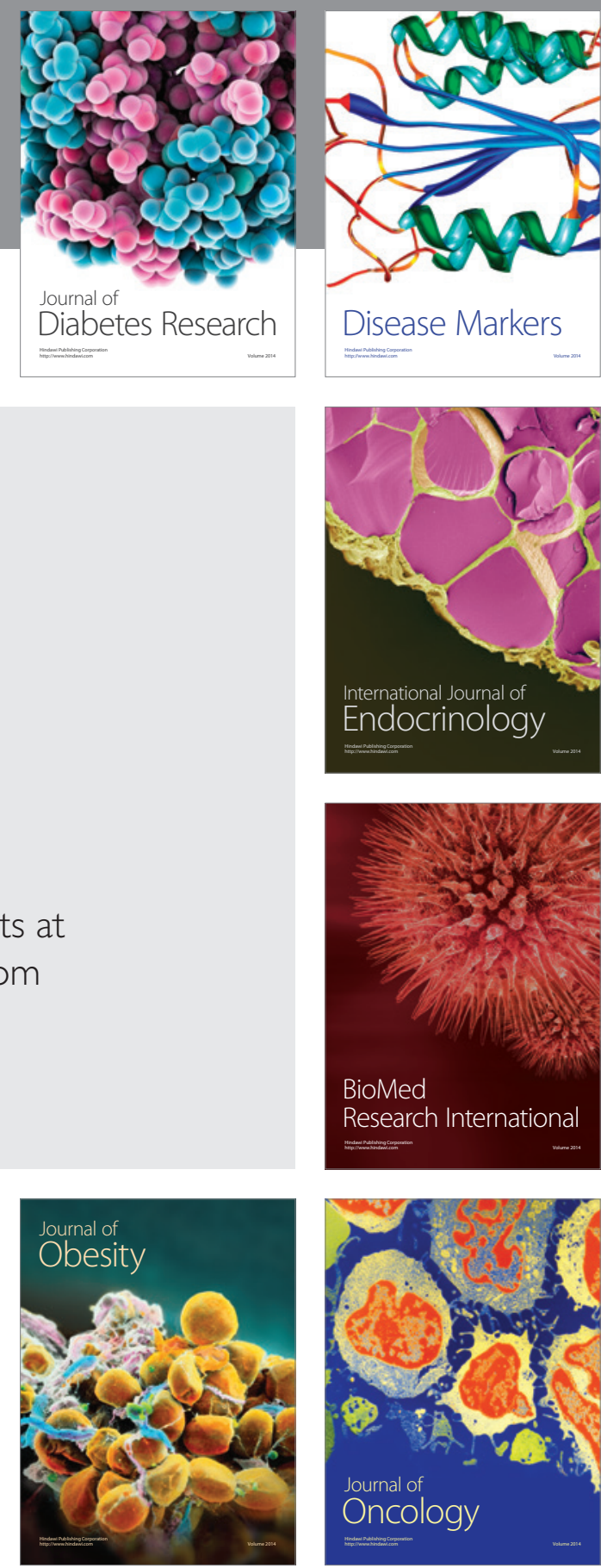

Disease Markers
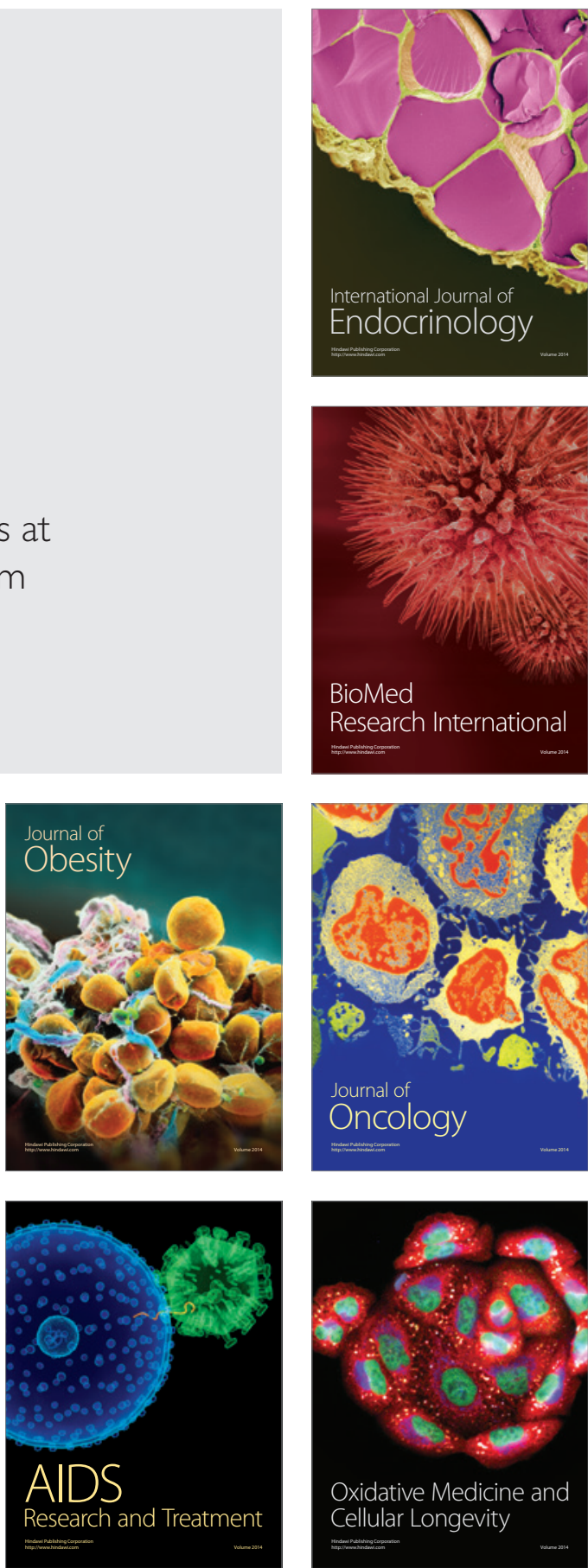